\title{
PARAMETERS FOR FUCHSIAN GROUPS II: TOPOLOGICAL TYPE $(1,1)$
}

\author{
Bernard Maskit
}

This note is an exploration of the space of Riemann surfaces of topological type $(1,1)$; that is, the space of distinct conformal structures on a torus with one boundary component; similar explorations for the space of Riemann surfaces of genus zero with four boundary components were considered in [M2]. There are several different approaches to this space. There is a general deformation space of a Riemann surface with boundary, marked with a basis for the fundamental group; these have been explored by Bers and Gardiner [B-G]. Another general approach is to look at canonical domains on closed surfaces; this was explored in [M3]. There are also explicit constructions due to Rosenberger [R] and Keen [K], using the known presentation of the modular group. In this paper, we give a similar explicit parametrization of the space of standard (and normalized) generators of Fuchsian groups representing tori with one boundary component. Our parametrization is intrinsic in the sense that it does not depend on a choice of base point.

Our parameter space has an interior and a boundary, where the interior consists of Riemann surfaces with holes (i.e., surfaces where the boundary is a deleted disc), and the boundary consists of Riemann surfaces with punctures (i.e., those where the boundary is a deleted point).

Our parameters have the following properties: after appropriate normalization, the entries in the matrices of the generators are explicit polynomials in the parameters (the matrices have positive, but not necessarily unit, determinant); the parameter space is a product of half-planes; and one of the parameters, $\lambda$, is the multiplier of the commutator. Since the commutator is parabolic if and only if the group represents a torus with a puncture, as opposed to a hole, we have $\lambda \geq 1$, and $\lambda=1$ if and only if the group represents a torus with a puncture. These parameters thus yield a real analytic structure on the space of marked Fuchsian groups of type $(1,1)$. This real-analytic structure is equivalent to the usual one in both the interior and on the boundary of our space. More precisely, the interior is real analytically equivalent to the reduced Teichmüller space (see Earle [E]) of tori with one hole, and the boundary is real analytically equivalent to the usual Teichmüller space of tori with one puncture.

Research supported in part by National Science Foundation Grant No. DMS 8701774.

doi:10.5186/aasfm.1989.1419 
We also remark that if we permit these parameters to become complex, while keeping $|\lambda|>1$, or by keeping $\lambda=1$, we obtain a complex analytic parametrization of the corresponding space of marked quasifuchsian groups; in these cases however, we have almost no information about the boundary of the corresponding parameter spaces.

In order to go from the parameter space, which is essentially a deformation space of Fuchsian groups, to the underlying space of Riemann surfaces, we need to define a fundamental domain for the action of the Teichmüller modular group. We do this in several steps.

If $S$ is a Riemann surface of type $(1,1)$, then generically there is a shortest non-dividing geodesic on $S$, call it $a$. There is also generically a shortest simple geodesic $b$ crossing $a$ exactly once. The corresponding elements $A$ and $B$ of the Fuchsian group generate it; we call these modular generators. They are generically well defined up to orientation; we show below that, up to orientation, every Riemann surface of topological type $(1,1)$ has at most two sets of modular generators.

The modular generators are defined by infinitely many inequalities; we show that they can be defined by just three. In order to state these inequalitites, we make the following definition. If

$$
M=\left(\begin{array}{ll}
a & b \\
c & d
\end{array}\right)
$$

is any element of $\operatorname{PGL}(2, \mathbf{R})^{+}$, the space of real $2 \times 2$ matrices with positive determinant, then we define the normalized trace function $T(M)$ by

$$
T(M)=|a+d| /(a d-b c)^{1 / 2} .
$$

In terms of this function, the modular generators can be defined by the inequalities $T(A) \leq T(B), T(B) \leq T(A B)$, and $T(B) \leq T\left(A B^{-1}\right)$.

These inequalities define a fundamental domain for action of the Teichmüller modular group that is very closely related to the usual fundamental domain for the action of the elliptic modular group. That is, the usual fundamental domain for the elliptic modular group is defined by the inequalities $|\tau| \geq 1$ and $-\frac{1}{2} \leq \operatorname{Re}(\tau) \leq \frac{1}{2}$. An equivalent set of inequalities is: $|\tau| \geq 1,|\tau| \leq|1+\tau|$, and $|\tau| \leq|1-\tau|$; that is, the shortest side has length one, and the sides of the parallelogram formed by 1 and $\tau$ are both shorter than both diagonals. A third equivalent definition is: $|\tau| \geq 1$, and for every $n, m \leq \mathbf{Z}$ with $m \neq 0,|n+m \tau| \geq|\tau|$.

In the final section, we translate these inequalities into our parameters, so as to again define the space of Riemann surfaces of type $(1,1)$. We write down the identifications of the sides, and we show that the resulting space is a product of an interval and a space homeomorphic to $\mathbf{H}^{2} / \operatorname{PSL}(2, \mathbf{Z})$; this agrees with the result in [M3]. 
1. In this section we define our parameters. We start with any point $x>1$, and draw four hyperbolic lines in the upper half-plane, $\mathbf{H}^{2}$, as follows. The line with endpoints at 0 and $\infty$ is called $S_{1}$; the line with endpoints at 0 and 1 is called $T_{2}$, the line with endpoints at 1 and $x$ is called $T_{1}$, and the line with endpoints at $x$ and $\infty$ is called $S_{2}$ (see Figure 1).

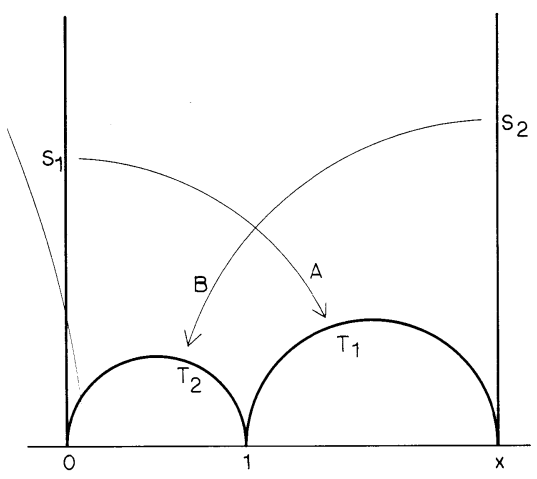

Figure 1.

Define

$$
\hat{A}=\hat{A}(x)=\left(\begin{array}{cc}
x & x \\
1 & x
\end{array}\right), \quad \hat{B}=\hat{B}(x)=\left(\begin{array}{cc}
0 & x \\
-1 & 2 x
\end{array}\right)
$$

and observe that for every $x, \hat{A}$ maps $S_{1}$ onto $T_{1}$, and $\hat{B}$ maps $S_{2}$ onto $T_{2}$. Let $\hat{G}=\hat{G}(x)=\langle\hat{A}, \hat{B}\rangle$, the group generated by $\hat{A}$ and $\hat{B}$, and let $D$ be the region bounded by the four lines, $S_{1}, T_{1}, S_{2}$, and $T_{2}$. Observe that $\hat{A}$ and $\hat{B}$ both map $D$ onto regions that are disjoint from $D$. Let $B_{1}$ be the open region in $\mathbf{H}^{2}$ between $S_{1}$ and $T_{1}$, and let $B_{2}$ be the open region in $\mathbf{H}^{2}$ between $S_{2}$ and $T_{2}$. Then $B_{1} \cup B_{2}=\mathbf{H}^{2}$, and $B_{1} \cap B_{2}=D \neq \emptyset$. It follows from Klein's combination theorem [M1, p. 139] that $\hat{G}$ is discrete, $\hat{G}=\langle\hat{A}\rangle *\langle\hat{B}\rangle$, and no-trivial translate of $D$ overlaps $D$. An easy computation also shows that the commutator $\hat{A}^{-1} \hat{B}^{-1} \hat{A} \hat{B}=\left[\hat{A}^{-1}, \hat{B}^{-1}\right]$ is parabolic with fixed point at $\infty$. Knowing this, we can also use Poincaré's polygon theorem to reach the above conclusions, and also to conclude that the interior of $D$ is a fundamental polygon for $\hat{G}$.

The general transformation mapping $S_{1}$ onto $T_{1}$, and mapping $D$ onto a region disjoint from itself, is then of the form $A=\hat{A} M$, where $M$ is a hyperbolic transformation, or the identity, with axis $S_{1}$. Similarly, the general transformation mapping $S_{2}$ onto $T_{2}$, while mapping $D$ onto a region disjoint from itself, is of the form $B=\hat{B} N$, where $N$ is a hyperbolic transformation, or the identity, with axis $S_{2}$. we write

$$
M=\left(\begin{array}{cc}
t & 0 \\
0 & 1
\end{array}\right), \quad N=\left(\begin{array}{cc}
s & x(1-s) \\
0 & 1
\end{array}\right)
$$


where $s$ and $t$ are both positive. We then obtain the general transformations:

$$
A=\hat{A} M=\left(\begin{array}{cc}
t x & x \\
t & x
\end{array}\right), \quad B=\hat{B} N=\left(\begin{array}{cc}
0 & x \\
-s & x(1+s)
\end{array}\right) .
$$

Using Klein's combination theorem again, we see that for any choice of parameters $s>0, t>0$, and $x>1$, the group $G=G(x, s, t)=\langle A, B\rangle$ is discrete, $G=\langle A\rangle *\langle B\rangle$, and no non-trivial translate of $D$ intersects $D$. An easy computation shows that $T\left(\left[A^{-1}, B^{-1}\right]\right)=s / t+t / s$; in particular, the commutator $\left[A^{-1}, B^{-1}\right]$ is parabolic if and only if $s=t$. In any case, $\left[A^{-1}, B^{-1}\right]$ has a fixed point at $\infty$; if $\left[A^{-1}, B^{-1}\right]$ is hyperbolic, then $\infty$ is the repelling fixed point if and only if $t>s$. We now set $t=\lambda s$, and define the parameter space:

$$
P=\left\{(x, s, \lambda) \in \mathbf{R}^{3} \mid x>1, s>0, \lambda \geq 1\right\} .
$$

If $\lambda=1$, then we can use Poincaré's theorem to conclude that $D$ is a fundamental polygon for $G$; hence, in this case $\mathbf{H}^{2} / G$ is a torus with one puncture. If $\lambda>1$, then $A$ and $B$ are hyperbolic and freely generate the discrete group $G$; the axes of $A$ and $B$ intersect; and the commutator is hyperbolic. Hence, in this case, $\mathbf{H}^{2} / G$ is a torus with one hole.

We also note that the generators $A$ and $B$ are oriented in the reverse of the usual "standard" set of generators. That is, if we renormalize so that the attracting fixed point of $A$ is at $\infty$, with the repelling fixed point at 0 , then the attracting fixed point of $B$ will be positive, and the repelling fixed point negative.

2. Now that we have our parameters, we define the abstract space they parametrize; we follow the development of Bers [B]. We start with some $G_{0}$ corresponding to a point in our space $P$; for definiteness, we set $x=2, s=1$, and $\lambda=1$. With this choice, $G_{0}$ represents the punctured square torus (see Section 8). A deformation of $G_{0}$ is a monomorphism $\psi: G_{0} \rightarrow \operatorname{PSL}(2, \mathbf{R})$ for which there is an orientation preserving homeomorphism $\varphi: \mathbf{H}^{2} \rightarrow \mathbf{H}^{2}$, with $\varphi \circ g \circ \varphi^{-1}(z)=\psi(z)$, for all $g \in G_{0}$, and for all $z \in \mathbf{H}^{2}$. Two deformations $\psi_{1}$ and $\psi_{2}$ are equivalent if there is an element $C$ in $\operatorname{PSL}(2, \mathbf{R})$ with $\psi_{1}(g)=C \psi_{2}(g) C^{-1}$ for all $g \in G_{0}$. The set of equivalence classes is the deformation space $\mathbf{F}=\mathbf{F}\left(G_{0}\right)$; it has a natural real analytic structure as a subset of $\operatorname{Hom}\left(G_{0}, \operatorname{PSL}(2, \mathbf{R})\right)$ modulo conjugation.

An element $\psi \in \mathbf{F}$ need not be type-preserving; that is, we might have that $\psi\left(\left[A^{-1}, B^{-1}\right]\right)$ is hyperbolic even though $\left[A^{-1}, B^{-1}\right]$ is parabolic.

In order to simultaneously normalize the groups $\psi\left(G_{0}\right)$, for $\psi \in F$, we need to observe that the points 0,1 , and $\infty$ are all conjugate parabolic fixed points in $G_{0}$. We simultaneously normalize by requiring that $\psi\left(\left[A^{-1}, B^{-1}\right]\right)$ have its (repelling) fixed point at $\infty$; that $\psi\left(\left[B, A^{-1}\right]\right)$ have its (repelling) fixed point at 0 , and that $\psi([A, B])$ have its (repelling) fixed point at 1 .

We make the observation that a deformation necessarily preserves the sense of each parabolic or hyperbolic transformation. To make this clearer, let $\psi$ be a 
deformation, normalized as above. Since $\left[A^{-1}, B^{-1}\right]$ is of the form $z \rightarrow z-\alpha$, $\alpha>0$, then $\psi\left(\left[A^{-1}, B^{-1}\right]\right)$ is of the same form if it is parabolic, while if it is hyperbolic, then, since its repelling fixed point is at $\infty$, and there are other fixed points normalized at 0 and 1 , its attractive fixed point is necessarily negative.

We will also need the (Teichmüller) modular group, defined as follows. A deformation $\psi$ is called modular if $\psi\left(G_{0}\right)=G_{0}$. The group of modular deformations acts on $\mathbf{F}$ by right multiplication; that is, if $\psi_{1}$ is any deformation, then $\psi\left(\psi_{1}\right)=\psi_{1} \circ \psi^{-1}$. The modular group, $\mathbf{M}$, is the effective part of this action. The quotient $\mathbf{F} / \mathbf{M}=\mathbf{R}$ is the moduli space, or Riemann space, of conformally distinct Riemann surfaces of genus 1 , with one boundary component.

3. There is an obvious map $\Phi: P \rightarrow \mathbf{F}$, given by the fact that every point in $P$ corresponds to a pair of generators of a Fuchsian group representing a torus with one boundary component, where the axes of these generators always intersect with the same orientation.

If $\psi$ is any deformation in $\mathbf{F}$, as normalized above, then we can read off $\Phi^{-1}(\psi)$ as follows: $x$ is the (repelling) fixed point of $[\psi(B), \psi(A)], s=(1-$ $\psi(B)(0)) / \psi(B)(0)$, and $\lambda$ is the positive square root of the multiplier of the commutator $\left[\psi\left(A^{-1}\right), \psi\left(B^{-1}\right)\right]$, where the multiplier is chosen to be $\geq 1(\lambda=1$, if the commutator is parabolic). Since $\psi$ is induced by an orientation preserving homeomorphism of $\mathbf{H}^{2}$, the fixed points of the elements of $G_{0}$ and $\psi\left(G_{0}\right)$ occur in the same order on the circle at infinity; in particular, $0<\psi(B)(0)<1$. It follows that $s>0$, and $x>1$.

We note incidentally that these three parameters can be defined independent of normalization. We already have $\lambda$ defined as the multiplier of the commutator; we can also express $x$ and $s$ in terms of cross ratios of fixed points of elements of $G$. More precisely,

$$
x=\left(r\left(\psi\left[B, A^{-1}\right]\right), r\left(\psi\left[A^{-1}, B^{-1}\right]\right) ; r\left(\psi\left[B^{-1}, A\right]\right), r(\psi[A, B])\right),
$$

and

$$
s=-\left(r\left(\psi\left[B, A^{-1}\right]\right), r(\psi[A, B]) ; r\left(\psi\left[A^{-1}, B^{-1}\right]\right), r\left(\psi\left(B\left[B, A^{-1}\right] B^{-1}\right)\right)\right),
$$

where $r(X)$ is the (repelling) fixed point of $X$, and the cross ratio $(0, \infty ; z, 1)=z$.

4. Our next goal is to find a fundamental domain for the action of the modular group $\mathbf{M}$ in its action on $P$. We start with a general construction.

Several facts about the topology of simple loops on a torus with one boundary component are used in what follows. They are all easily derivable by looking either at a fundamental domain for a torus with a puncture, such as Figure 1, or by looking at the unit square with its sides identified in the usual fashion, and a hole cut out of its middle, as in Figure 2.

We start by defining a set of inequalities for closed geodesics that pick out a marking on each Riemann surface of topological type $(1,1)$. This marking is 


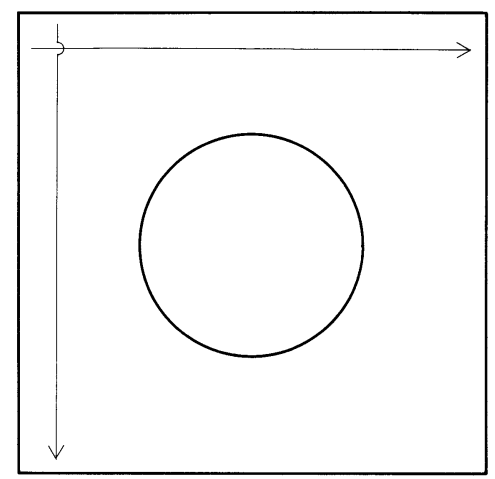

Figure 2.

generically unique up to the (hyper)elliptic involution, which acts as the identity on $\mathbf{F}$. That is, for each such Riemann surface, we pick out a generically unique point in $\mathbf{F}$ representing the given surface.

Let $S$ be a Riemann surface of topological type $(1,1)$, which we regard as being endowed with the usual hyperbolic metric. Let $w$ be the shortest closed nondividing geodesic on $S$; if the shortest geodesic is not unique, pick one such. Let $v$ be the shortest simple closed geodesic which croses $w$ at exactly one point. Again, if necessary, make some choice of the shortest such geodesic. It is well known, and we will see below that $w$ and $v$ are generically unique up to orientation. Obviously we can not tell the difference between $w$ and its inverse, or between $v$ and its inverse. Make some choice of orientation for $w$; then choose the orientation on $v$ so that going around the point of intersection in the positive direction, starting at the tail of $w$, we first meet the head of $v$, then the head of $w$, then the tail of $v$, and then come back to the tail of $w$.

Let $x_{0}$ be the point of intersection of $v$ and $w$, and let $A, B$, be the element of $\pi_{1}\left(S, x_{0}\right)$ represented respectively by $w, v$. Then $A$ and $B$ generate $\pi_{1}\left(S, x_{0}\right)$, which we now consider to be a Fuchsian group generated by $A$ and $B$, where $A$ and $B$ are normalized as above. Then, once we have made our choices, if necessary, of shortest geodesics, $A$ and $B$ are uniquely determined, except that we can replace $A$ by $A^{-1}$ and $B$ by $B^{-1}$. As is well known, this replacement corresponds to the (hyper)elliptic involution on $S_{0}$, which acts ineffectively on $\mathbf{F}$ (it is an easy computation using our parameters to check that the element of $\mathbf{M}$ taking $(A, B)$ into $\left(A^{-1}, B^{-1}\right)$, acts as the identity on $\left.P\right)$.

Two hyperbolic elements $X$ and $Y$ of a Fuchsian group $G$, representing a torus with one boundary component, are called standard generators if they are both primitive (i.e., neither is a non-trivial power of any other element of $G$ ), their axes intersect, and their axes both project to simple loops on $\mathbf{H}^{2} / G$; it is well known that any such pair of elements do indeed generate $G$. 
Theorem. Let $A$ and $B$ be standard generators for the Fuchsian group $G$ of topological type $(1,1)$. Suppose that $T(A) \leq T(B), T(B) \leq T(A B)$, and $T(B) \leq T\left(A^{-1} B\right)$. Then

(i) if $Z$ is any element of $G$, where $Z$ is not a commutator, $T(Z) \geq T(A)$;

(ii) if there is a $Z \in G, Z \neq A^{ \pm 1}$, where $Z$ is not a commutator, and $T(Z)=$ $T(A)$, then $T(B)=T(A)$; and either $Z$ or $Z^{-1}$ is conjugate to $B$ or $A B$ or $A B^{-1}$

(iii) for any $Z \in G$, where $A$ and $Z$ are standard generators, $T(Z) \geq T(B)$; and (iv) if there is an element $Z \in G$, where $A$ and $Z$ are standard generators, with $T(Z)=T(B)$, then either $Z$ or $Z^{-1}$ is conjugate to either $B$ or $A B$ or $A B^{-1}$.

Before going on to the proof of this theorem, we remark that parts (iii) and (iv) are well known; they were proven by Rosenberger [R].

Proof. Let $u, v$ be the projection of the axis of $A, B$, respectively; by assumption $u$ and $v$ intersect at exactly one point. We first prove part (iii). We temporarily assume that the boundary component of $S=\mathbf{H}^{2} / G$ is a puncture so that we can use Figure 1 as a fundamental domain for $G$. Let $L$ be the axis of $Z$, and let $L^{\prime}$ be $L$, translated in segments so that it lies entirely in $D$; that is, $L^{\prime}$ is a union of (hyperbolic) line segments all lying in $\bar{D}$. Since the projection of $L$ crosses $u$ exactly once, $L^{\prime}$ has exactly one endpoint on $S_{2}$, and exactly one endpoint on $T_{2}$. It follows that $Z$ is conjugate to a transformation either of the form $A^{m} B$, or of the form $A^{m} B^{-1}$.

Since the above argument is purely topological, it also holds in the case that $\mathbf{H}^{2} / G$ has a hole.

It was first observed by Kerckhoff $[\mathrm{Ke}]$ that $T\left(X^{n} Y\right)$ is a convex function of $n$. Since the result in this special case, where $X$ and $Y$ are hyperbolic with intersecting axes, is quite simple, we include the proof. Normalize so that $X(z)=$ $\lambda^{x} z, \lambda>0$, and write $Y(z)=(a z+b) /(c z+d), a d-b c=1$. Then, since the product of the fixed points of $Y$ is negative, $a$ and $d$ have the same sign. For purposes of calculation, we can choose $a$ and $d$ to be both positive. An easy calculation now shows that $T\left(X^{x} Y\right)$ is a strictly convex function of $x$. Since we know that $T(A B) \geq T(B)$, and $T\left(A^{-1} B\right) \geq T(B)$, it follows that for all $n \in \mathbf{Z}$ with $|n|>1, T\left(A^{n} B\right)>T(B)$. This proves statements (iii) and (iv).

We turn now to parts (i) and (ii); we assume that there is a $Z \in G$, with $Z \neq A^{ \pm 1}$, and $Z$ not a commutator, with $T(Z) \leq T(A)$. Observe first that if there is a closed geodesic, whose homotopy class is not in the commutator subgroup, and which is shorter than $u$, then there is a simple closed geodesic with the same properties. Hence we can assume that $t$, the projection of the axis of $Z$, is simple.

If we cut $S=\mathbf{H}^{2} / G$ along $u$, we are left with a sphere with three boundary components; hence either $t$ is a power of $u$ or $u^{-1}$, or $t$ is not simple, or $t$ crosses $u$. Hence we can assume that $t$ is simple and crosses $u$. Since we have already taken care of the case that $t$ and $u$ cross exactly once, we can assume that the 
number of crossings is $n \geq 2$. By looking at the square with a hole cut out of its middle, it is easy to see that since $t$ is simple, all the crossings of $u$ occur in the same direction. We pick one of these crossings as the base point, and label the crossings as $x_{1}, \ldots, x_{n}$, in the order they appear as we traverse $t$. These $n$ points divide $t$ into $n$ arcs; call them $t_{1}$, starting at $x_{1}, t_{2}$, starting at $x_{2}$, and so on up to $t_{n}$ which ends at the base point $x_{1}$.

For each successive pair of crossings $x_{m}, x_{m+1}$, let $u_{m}$ be the shorter of the two arcs of $u$ between $x_{m}$ and $x_{m+1}$. Orient $u_{m}$ so that it starts at $x_{m+1}$ and ends at $x_{m}$. If the two arcs have equal length, then choose either of them as $u_{m}$. Since crossings of $t$ and $u$ are either all positive or all negative, every loop in the homotopy class of $t_{m} u_{m}$ crosses $u$, and the shortest geodesic, $v_{m}$, freely homotopic to $t_{m} u_{m}$, crosses $u$ exactly once. Since $t_{m} u_{m}$ is not a geodesic, $\left|v_{m}\right|<\left|t_{m} u_{m}\right|$, where we use $|x|$ to denote the (hyperbolic) length of $x$. By part (iii) of this theorem, $\left|v_{m}\right| \geq|v|$. We now have

$$
\sum\left|t_{m} u_{m}\right|>\sum\left|v_{m}\right| \geq n|v| \text {. }
$$

On the other hand

$$
\sum\left|t_{m} u_{m}\right|=\sum\left|t_{m}\right|+\sum\left|u_{m}\right| \leq|t|+\frac{1}{2} n|u| .
$$

Combining (1) and (2), we obtain

$$
|t|>n|v|-\frac{1}{2} n|u|=\frac{1}{2} n|v|+\frac{1}{2} n(|v|-|u|) \geq \frac{1}{2} n|v| \geq|v| .
$$

We have shown that every simple geodesic which crosses the projection of the axis of $A$ at least twice is strictly longer than $v$, the projection of the axis of $B$. This is part (i) of our theorem; part (ii) follows at once from the strict inequality in (3), together with the strict convexity of $T\left(A^{n} B\right)$.

We restate the same theorem in terms of geodesics.

Corollary. Let $S$ be a hyperbolic torus with one boundary component. Suppose $u$ and $v$ are simple geodesics on $S$, where $u$ and $v$ cross at exactly one point. Let $w_{1}$ be the shortest geodesic freely homotopic to $u v$, and let $w_{2}$ be the shortest geodesic freely homotopic to $u v^{-1}$. Suppose that $|u| \leq|v|,|v| \leq\left|w_{1}\right|$ and $|v| \leq\left|w_{2}\right|$. Then

(i) no non-dividing geodesic on $S$ is shorter than $u$;

(ii) if there is a non-dividing geodesic $t$ on $S$ with the same length as $u$, then $|v|=|u|$, and up to base point and orientation, $t=u$, or $t=w_{1}$ or $t=w_{2}$.

(iii) if $t$ is a non-dividing simple geodesic on $S$ crossing $u$ exactly once, then $|t| \geq|v| ;$ and

(iv) if there is a non-dividing simple geodesic $t$ on $S$ crossing $u$ exactly once, with $|t|=|v|$, then, up to base point and orientation, $t=v$, or $t=w_{1}$ or $t=w_{2}$. 
5. The results of the theorem above, together with our choice of $A$ and $B$, can be summarized as follows. Except for the possibility of replacing $A$ by $A^{-1}$ and $B$ by $B^{-1}$, a fundamental domain for the action of the modular group $\mathbf{M}$ on $P$ can be realized by the inequalities:

$$
T(A) \leq T(B), \quad T(B) \leq T(A B), \quad \text { and } \quad T(B) \leq T\left(A B^{-1}\right),
$$

for these inequalities guarantee that no geodesic, except perhaps for the commutator, is shorter than $u$, the projection of the axis of $A$; also that of all simple geodesics crossing $u$ exactly once, none is shorter than $v$, the projection of the axis of $B$.

We can write the inequalities (4) in terms of the parameters $x, s$, and $\lambda$. This yields the following expressions, in the same order as the inequalities in (4):

$$
\begin{aligned}
x & \geq 1+(1+\lambda s)^{2} / \lambda(1+s)^{2} \\
(1-\lambda s)(1+s)^{2} x^{2}+(1+s) s(3 \lambda-2+\lambda s) x+s^{2}(\lambda-1)^{2} & \geq 0 \\
\lambda(\lambda s-1)(1+s)^{2} x^{2}+\lambda(1+s)(1+3 s-2 \lambda s) x+s(\lambda-1)^{2} & \geq 0 .
\end{aligned}
$$

Equality in each of these three inequalities determines a side of the fundamental domain; call the sides in order: $S_{1}, S_{2}$, and $T_{2}$.

One can read off from (4) that $\alpha(A, B)=\left(B, A^{-1}\right)$ maps $S_{1}$ onto itself, and that $\beta(A, B)=(A, B A)$ maps $S_{2}$ onto $T_{2}$. It is clear that these generate $\mathbf{M}$; one also easily sees that they satisfy the relations: $\alpha^{2}=(\alpha \beta)^{3}=1$. In terms of our parameters, these generators are given by

$$
\alpha(x, s, \lambda)=(x /(x-1), 1 / \lambda s, \lambda),
$$

and

$$
\beta(x, s, \lambda)=((x+\lambda s) / \lambda s, s(x-1) /(x+\lambda s), \lambda) .
$$

Equality in the first inequality of $(5)$ says that $T(A)=T(B)$; it is not hard to see that this is equivalent to the statement that there is a (hyperbolic) reflection $R$ so that conjugation by $R$ interchanges $A$ and $B$.

It was remarked by Bers [B] that the set of Beltrami differentials supported on a disc sweeps out a neighborhood in Teichmüller space, so every torus with a hole can be conformally embedded in many closed tori. However, if we regard a torus with a hole as being canonically embedded in a closed torus as a circle domain (see Strebel [S] and [M3]), then one easily sees that every conformal (including orientation reversing) automorphism of a torus with a hole extends to a conformal automorphism of the corresponding closed torus, and conversely, every automorphism of finite order of the closed torus is conjugate (in the group of all automorphisms of the closed torus) to one that keeps invariant the embedded torus 
with a hole. It follows that we can identify the set of tori with one boundary component, where $T(A)=T(B)$, as being the same, under the canonical embedding, as the set of closed tori with $|\tau|=1$.

Similarly, equality for the middle inequality asserts that there is a reflection $R$ so that conjugation by $R$ interchanges $A$ and $A B$. This is equivalent, again using the canonical embedding, to the statement that $\operatorname{Re}(\tau)=-\frac{1}{2}$. Likewise, equality in the bottom inequality corresponds to $\operatorname{Re}(\tau)=\frac{1}{2}$.

One can also find the set corresponding to the set $\operatorname{Re}(\tau)=0$. A point $(x, s, \lambda)$ in $P$ corresponds to such a $\tau$ if and only if there is a reflection $R$ conjugating $B$ into itself and $A$ into its inverse. If we write the fixed points of $B$ as $a$ and $b$, then it is easy to see that the reflection (see [M2])

$$
R=\left(\begin{array}{cc}
s & -2 p \\
p & -s
\end{array}\right)
$$

where $s=a+b$ and $p=a b$, conjugates $B$ into itself; that is, the fixed point set of $R$ is the axis of $B$. An easy computation shows that $R$ conjugates $A$ into $A^{-1}$ if and only if

$$
(\lambda s-1)(s+1) x=2 s(\lambda-1) .
$$

We remark that the above reduces to the equation $s=1$ on the boundary of our space; that is, when $\lambda=1$.

7. It was shown in [M3] that the moduli space of tori with one hole is real analytically equivalent to the product of an interval and $\mathbf{H}^{2} / \operatorname{PSL}(2, \mathbf{Z})$. We can explicitly realize this correspondence with our parameters as follows. We already have our parameter space $P$ written as a product, where each slice $\lambda=$ constant is kept invariant by the full modular group. Hence our representation of moduli space already is a product. It is easy to see that we cannot have simultaneous equality in the bottom two inequalities of (5). If we have equality in the top two inequalities, then we can solve the first for $x$; this yields the following polynomial:

$$
\begin{aligned}
\lambda^{4}(\lambda+1) s^{5} & +2\left(\lambda^{4}+\lambda^{3}\right) s^{4}-\left(3 \lambda^{4}+2 \lambda^{3}-\lambda^{2}\right) s^{3} \\
& -\left(\lambda^{4}+8 \lambda^{3}+7 \lambda^{2}\right) s^{2}-\left(2 \lambda^{3}+7 \lambda^{2}+5 \lambda\right) s-(\lambda+1)^{2}=0 .
\end{aligned}
$$

By Descarte's rule of signs, this has exactly one positive root for each $\lambda \geq 1$.

Similarly, if we have equality in the top and bottom inequalities, we solve the top for $x$, write out the bottom as a polynomial, and obtain:

$$
\begin{aligned}
\lambda^{3}(\lambda+1)^{2} s^{5} & +\left(5 \lambda^{4}+7 \lambda^{3}+2 \lambda^{2}\right) s^{4}+\left(7 \lambda^{3}+8 \lambda^{2}+\lambda\right) s^{3} \\
& -\left(\lambda^{3}-2 \lambda^{2}-3 \lambda\right) s^{2}-2\left(\lambda^{2}+\lambda\right) s-(\lambda+1)=0,
\end{aligned}
$$

which also has exactly one positive root for each $\lambda \geq 1$. It is now clear that this representation of the moduli space of tori with one boundary component is a (real analytic) product of a half-open interval $(1 \leq \lambda<\infty)$ and $\mathbf{H}^{2} / \operatorname{PSL}(2, \mathbf{Z})$. 
8. The inequalities in (5) are somewhat unpleasant. However, they become significantly nicer in the special case of groups representing tori with punctures; that is, when $\lambda=1$. In this case, they reduce to:

$$
x \geq 2, \quad s \leq x /(x-1), \quad s \geq(x-1) / x .
$$

We can now easily read off from these that $x=2$ corresponds to $|\tau|=1 ; s=$ $x /(x-1)$ corresponds to $\operatorname{Re}(\tau)=-\frac{1}{2} ; s=(x-1) / x$ corresponds to $\operatorname{Re}(\tau)=\frac{1}{2}$; the square $\tau=i$ is given by $(x, s)=(2,1)$; the rhombus $\tau=(1+\sqrt{3}) / 2$ corresponds to $(x, s)=\left(2, \frac{1}{2}\right)$. We have already remarked that the line $\operatorname{Re}(\tau)=0$ corresponds to the line $s=1$.

\section{References}

[B] Bers, L.: Uniformization, moduli, and Kleinian groups. - Bull. London Math. Soc. 4, 1972, 257-300.

[B-G] Bers, L., and F. Gardiner: Fricke spaces. - Mathematical Sciences Research Institute preprint, 1985.

[E] EARLe, C.J.: Reduced Teichmüller spaces. - Trans. Amer. Math. Soc. 126, 1967, 54-63.

[K] KeEN, L.: On fundamental domains and the Teichmüller modular group. - Contributions to Analysis, Academic Press, New York-London, 1974, 185-194.

[Ke] Kerckhoff, S.: The Nielsen realization problem. - Ann. of Math. (2) 117, 1983, 235-265.

[M1] MASkit, B.: Kleinian groups. - Springer-Verlag, New York-Berlin-Heidelberg, 1988.

[M2] MAskit, B.: Parameters for Fuchsian groups I - Signature $(0,4)$. - Holomorphic Functions and Moduli, II, p. 251-265. Math. Sci. Res. Inst. Pub. 11. Springer-Verlag, New York, 1988.

[M3] Maskit, B.: Canonical domains on Riemann surfaces. - Proc. Amer. Math. Soc. (to appear).

[R] Rosenberger, G.: Fuchssche Gruppen, die freies Produkt zweier zyklischer Gruppen sind, und die Gleichung $x^{2}+y^{2}+z^{2}=x y z$. - Math. Ann. 199, 1972, 213-227.

[S] Strebel, K.: Ein Klassifizierungsproblem für Riemannsche Flächen vom Geschlecht 1. Arch. Math. 48, 1987, 77-81.

State University of New York at Stony Brook

Department of Mathematics

Stony Brook, NY 11794-3651

U.S.A.

Received 30 May 1988 